\title{
25 Research Square \\ Epidemiological Characteristics of COVID-19 Deaths In China: An Analysis of Environmental Factors
}

\section{Xixi Feng}

Chengdu Medical College

Peipei Du

Chengdu Medical College

Guobao Li

Shenzhen Third People's Hospital

\section{Peihua Cao}

Southern Medical University

Jiaohua Luo

Army Medical University

Xiaodong Yan

Shandong University

Jiawei Luo

Sichuan University

Daihai He

The Hong Kong Polytechnic University

Lin Yang

The Hong Kong Polytechnic University

Xiaohui Wang

Lanzhou University

Shu Yang ( $\nabla$ yangshu@cdutcm.edu.cn )

Chengdu University of Traditional Chinese Medicine https://orcid.org/0000-0002-1859-6362

\section{Yang Fu}

Southern University of Science and Technology

\section{Yaolong Chen}

Lanzhou University

\section{Research article}

Keywords: COVID-19, Coronavirus, Death, Case fatality rate, Environmental factors

Posted Date: November 19th, 2021 
DOI: https://doi.org/10.21203/rs.3.rs-757532/v2

License: (c) (1) This work is licensed under a Creative Commons Attribution 4.0 International License. Read Full License 


\section{Abstract}

Background: As of April 2020, most of the confirmed cases outside Hubei province have been cured or confirmed dead in China. We aimed to understand environmental factors leading to COVID-19-related mortality in non-Hubei region.

Methods: We collected spatial-temporal and environmental data of 99 cases of COVID-19-related deaths outside of Hubei province in Mainland China between January 22, 2020 and April 6, 2020. A descriptive analysis, including a spatial-temporal distribution of daily reported diagnosed cases and related deaths, was conducted. We analyzed the possible environmental factors that affect the provincial-level case fatality rate (CFR) of COVID-19 outside Hubei, China.

Results: Among the 99 reported deaths, 59 (59.6\%) were male and 40 (40.4\%) were female. The mean age at death was 71.30 (SD 12.98) years and 74 deaths were among those 65 years or older. The CFR was negatively correlated with temperature $(r=-0.679, P<0.001)$ and humidity $(r=-0.607, P=0.002)$, while latitude was positively correlated with the CFR $(r=0.636, P=0.001)$. There were no statistically significant associations between CFR and the social environment factors.

Conclusion: Higher CFR of COVID-19 was associated with lower temperature, lower humidity, and higher latitude. Continual analysis of daily reported diagnoses and mortality data can help healthcare professionals and policy makers understand the trends within a country in order to better prepare nationwide prevention and care guidelines, along with adequately appropriate funds accordingly.

\section{Background}

Since the onset of the novel coronavirus disease 2019 (COVID-19) outbreak in Wuhan, capital city of Hubei province in China in December 2019, it has spread to 207 countries and regions around the world and directly resulted in more than 1,279,722 confirmed cases and 72,614 deaths [1]. In order to control the spread of the disease and its impact, China carried out dramatic non-pharmaceutical interventions across the country, including locking down cities and communities, closing schools and entertainment venues, implementing social distance and shelter in place policies, cancelling large gatherings and celebrations, restricting domestic and international travel, extending screening and testing, and keeping suspected cases and high risk groups in quarantine. As a matter of fact, these containment measures have successfully and rapidly curbed outbreak trends, especially in non-Hubei regions, where the newly confirmed COVID-19 cases have gradually decreased since early February, and the active cases has been kept in a relatively low level from late March. As of April 6, 2020, there were 80,757 confirmed cases, 76,882 patients recovered and discharged from the hospital, and 3,331 COVID-19 related deaths documented in mainland China, while 12,959 confirmed cases, 12,809 patients recovered and discharged, and 119 deaths were reported from outside Hubei [2]. This equates to a crude case fatality rate(CFR) of $4.33 \%$ in China and $0.92 \%$ in non-Hubei region. Actually, the crude CFRs range from $0.08 \%$ to $3.95 \%$ in provinces outside Hubei, however the reason hasn't been elucidated yet. At present, most of the 
investigations, which aim to understand the factors leading to COVID-19 related mortality, have focused on the description of biological factors, clinical characteristics, and laboratory indicators. Epidemic and clinical studies have currently shown that the main factors influencing COVID-19 CFR are age and comorbidities [3-5]. However, it's still difficult to explain the differences of CFRs among countries such as Italy, Spain, and German, where the age structure and the health level of the population are similar. Assessing the influencing factors of COVID-19 related deaths are crucial to determine the appropriate measures of prevention and control and to inform the health policy making. Factors that affect CFR are diverse, but few studies explored the effects of environmental factors that may lead to an increased risk of death. The environmental factors in this study refer to the natural environment factors such as temperature, humidity, and latitude, and social environment factors such as age structure, economic development, health service resources, which probably related to the CFR. Thus, in this study, based on publicly available data of COVID-19 cases and deaths from Health Commissions, as well as environmental factors of all provinces in mainland China except Hubei, we described the spatial-temporal distribution of COVID-19 related deaths and analyzed the possible environmental factors that affect the crude CFR outside of Hubei, China.

\section{Methods}

COVID-19 data were obtained from official websites and officially certified online media platforms of 30 provincial regions in mainland China, except Hubei Province, from March 15, 2020 to April 7, 2020. The confirmed case of COVID-19 was defined according to the "Diagnosis \& Treatment Scheme for Novel Coronavirus Pneumonia (Trial version 1 to 7)" enacted by the National Health Commission of China. According to the definition, a confirmed case is an individual who fulfills the criteria of a suspected case based on comprehensive analysis of epidemiological history and clinical manifestations, and has one of the following types of pathogenic evidence:1. Positive result by using real-time reverse-transcriptase polymerase-chain-reaction (RT-PCR) of respiratory specimens or blood specimens for detection of novel coronavirus nucleic acid; or 2. Respiratory specimens or blood specimens for viral gene sequencing, which are highly homologous with known novel coronaviruses; or 3. Positive result of specific novel coronavirus $\operatorname{lgM} / \mathrm{lgG}$ antibody, the novel coronavirus specific IgG antibody was positive from negative or 4 times higher than that in acute phase [6]. The COVID-19 related death was defined as fatal cases with laboratory-confirmed SARS-CoV-2 infection. The duration of deaths was defined as the interval between earliest date of symptom onset (i.e., cough, fever, or myalgia) and the date of confirmed death. The CFR in each province is calculated by dividing the number of cumulative deaths by the number of cumulative confirmed case, and was reported as percentage.

Data on environmental factors including natural environment factors and social environment factors were collected from the China National Statistical Yearbook [7]. As this was a retrospective analysis of reported case data, no informed consent was needed, no ethical issues with any analysis. The average air temperature, average humidity in February of each province, and the average altitude, average latitude of each provincial capital city was collected to study the effect of natural environment factors on CFR. 
Additionally, the social environment factors included in this study were per capita GDP, hospital beds per thousand population, health technicians per thousand population, medical practitioners per thousand population, proportion of the population over the age of 65 , and population density.

The basic characteristics and spatial-temporal distribution of death cases are reported as the percentages in different categories. Continuous variables are presented as mean and standard deviation (SD). Scatter plots were conducted to visualize the relationships between CFR and environmental factors. The association between the different environmental factors and the CFR was evaluated using Spearman's rank correlation coefficient. A p-value of $<0.05$ was considered as statistically significant. All data analysis was conducted using R version 3.6.1 (R Core Team (2013). R: A language and environment for statistical computing. R Foundation for Statistical Computing, Vienna, Austria. http://www.Rproject.org/).

\section{Results}

\section{Data collection and Demographic characteristics}

The first death outside of Hubei province in mainland China was reported on January 22, 2020, and 127 cumulative confirmed cases were reported on that day. As of April 6, 2020, there were a total of 12,959 confirmed cases and 119 death cases outside of Hubei. Among the 119 deaths, 99 had explicit demographic information and medical history. Of the 99 deaths, 59 (59.6\%) were male while $40(40.4 \%)$ were female. The average death age was 71.30 (SD 12.98) years, and was 69.20 (SD 14.60) years for male, 74.40 (SD 9.49) years for female respectively. 74 (74.7\%) deaths were among patients aged 65 years or older. The youngest death reported was a 25 -year old male while the oldest was a 94 -year old female. The average duration from onset of symptom to death was 9 days in these 99 deaths.

\section{Spatial-temporal distribution of death cases}

\section{Temporal distribution}

The COVID-19 daily new confirmed cases and deaths from January 22 to April 6 are shown in Figure 2 . As shown in Figure 2, the daily new confirmed cases outside Hubei gradually increased until reaching a peak of 890 cases on February 3, then gradually declined. The daily new deaths outside Hubei remained relatively low before February 5, then gradually increased and reached a peak of 12 cases on February 12 . Thereafter the curve continued downward, till there were less than three deaths per day after February 19. Interestingly, the peaked date of new deaths was exactly 9 days after the peaked date of new cases (February 3).

\section{Spatial distribution}

The first reported COVID-19-related death occurred in Hebei province on January 22, 2020. As of April 6, there were 119 deaths and 12,959 confirmed cases outside of Hubei province, resulting in a CFR of $0.90 \%$ outside Hubei. Of all provinces outside Hubei, Henan reported the highest number of COVID-19-related 
deaths $(n=22)$, as seen in Figure 3. Xinjiang, Hainan, and Heilongjiang had higher CFR, however, of 3.95\%, $3.57 \%$, and $2.70 \%$, respectively. In Xinjiang, the cumulatively confirmed cases were relatively small, with only 76 cases, and all three deaths in Xinjiang were from Xinjiang Production and Construction Corps. No deaths were reported in five provinces (Jiangsu, Ningxia, Qinghai, Shanxi, and Tibet).

\section{Analysis of influencing factors}

\section{Natural Environment Factors and CFR}

It can be seen from Figure 4 that air temperature is negatively correlated with CFR, which generally shows that the higher the temperature, the lower CFR $(r=-0.679, P<0.001)$. Humidity was also found to be negatively correlated with CFR $(r=-0.607, P=0.002)$, while latitude was positively correlated with CFR $(r=0.636, P=0.001)$ Hainan and Xinjiang were not included in the analysis, as they were considered outliers. The correlation between altitude and CFR were not statistically significant $(P>0.05)$.

\section{Social Environment Factors and CFR}

No statistically significant ( $P>0.05$ ) associations were found between CFR and the following provinciallevel social environment indicators, including per capita GDP, hospital beds per thousand population, health technicians per thousand population, medical practitioners per thousand population, proportion of the population over the age of 65 , or population density (Table 1 ).

\section{Discussion}

As of April 6,2020 in mainland China, there were a total of 12,959 confirmed cases and 119 deaths outside of Hubei, resulting in a crude CFR of $0.90 \%$. This CFR is lower than $5.14 \%(2,571 / 50,008)$ in Wuhan, China, $2.00 \%(73 / 3,654)$ in Japan, $1.81 \%(186 / 10,284)$ in South Korea, $2.72 \%(8,358 / 307,318)$ in the United States, and $12.32 \%(15,889 / 128,948)$ in Italy [1]. The success in controlling the epidemic and reducing CFR of COVID-19 patients outside of Hubei province in mainland China is remarkable. Our analysis of this phenomenon was attributed to the effective restrictive measures of the Chinese government and the relatively abundant medical resources in outside-Hubei areas. We performed descriptive analysis on the spatial-temporal distribution of all deaths outside of Hubei province. Prior to the implementation of Wuhan's lockdown policy on January 23, massive numbers of people moved across the country, especially to the surrounding provinces of Hubei, such as Henan and Hunan provinces. Daily new cases reported in Zhejiang and Guangdong provinces, which is respectively the commercial center of East China and South China and accumulate a great amount of mobile population, also showed a rapid growth trend similar to that of Wuhan. Daily new confirmed cases peaked at February 2, while daily new deaths peaked on February 12, 9 days after the peak of new cases. We speculated that the emergence of this interesting phenomenon might be related to the average duration of deaths, and the average duration from symptom onset to deaths among fatal cases was also 9 days in this study. Our result is shorter compared to another study reporting a duration of 10.56 days in Wuhan 
[8]. With adequate medical resources, the majority of deaths outside Hubei were cases of rapid onset and severe illness, which was likely to contribute to their shorter duration.

In this study, among the 99 confirmed COVID-19 related deaths, $59.6 \%$ were males, while $40.4 \%$ were female. Our results were consistent with an earlier study. According to an epidemiological survey of 72,314 COVID-19 patients published by the Chinese Centers for Disease Control and Prevention (China $\mathrm{CDC})$ in February 2020, the number of confirmed male cases was higher than that of females $(51.4 \%$ vs. 48.6\%) [9]. Taking into account the composition ratio of confirmed male and female cases, the male-tofemale CFR in this study was 1.43: 1, which meant that the risk of death for males with COVID-19 is 1.43 times of that for females. A recent article describing 113 deaths in Wuhan also showed that more males died than females (73.5\% vs. 26.5\%) [10]. Similar findings have been observed in several countries with severe COVID-19 epidemics, such as in Italy, where $70.2 \%$ of deaths are among males [11] and Spain, where $62.2 \%$ of deaths were among men [12]. South Korea, which has been touted for its epidemic response and vast number of tests, reported a somewhat similar distribution as to in our study, with $54.0 \%$ of deaths among men and $46.0 \%$ among women [13]. What is particularly noteworthy is that men died at a younger age due to COVID-19 compared to females (mean age: 69.20 vs. 74.69 years). Gender differences in smoking and the prevalence of lung diseases, diabetes, and hypertension (all of which are reportedly higher in men than in women) [14], may be a leading factor for these differences [15]. Associated with these medical conditions, men may have a higher risk of being in serious or critical condition, or even death, compared to women with COVID-19. The results show additionally that the majority of deaths were among those aged 65 years or older, which is consistent with another small-scale study in Wuhan [16]. Due to hypoimmunity or basic disease, elderly may be more susceptible to COVID19 , which potentially result in a higher risk of death [17].

According to our research, there is a significantly higher risk of death for COVID-19 infected patients living in regions of lower temperature, lower humidity, or higher latitude. Heilongjiang province, for example, is the northernmost and easternmost provincial administrative region in China, with five to six months of cold weather every year. The high prevalence of respiratory diseases such as tracheitis, emphysema, and cor pulmonale may be one of the reasons for the high CFR of COVID-19 in this region. A potential relationship between temperature, humidity and latitude and the survival and diffusion of Sars-Cov-2 has been proposed in another study [18], with COVID-19 spreading over an area roughly $30-50^{\circ} \mathrm{N}$ with an average temperature of $5-11^{\circ} \mathrm{C}$ and absolute humidity of $4-7 \mathrm{~g} / \mathrm{m}^{3}$. It was not yet possible to judge the pattern of COVID-19 CFR at higher temperature area, as the disease was prevalent mainly in February, when the maximum temperatures of most areas in China did not exceed $20^{\circ} \mathrm{C}$. But it's worth noting that areas with low temperatures, low humidity, and high latitudes should pay more attention to prevention and control and self-preservation when COVID-19 becomes prevalent again in the spring to reduce deaths, especially if COVID-19 is to coexist with us for a long time.

Lastly, we found that there was no evidence of correlation between CFR and social environment factors, such as proportion of the population aged 65 years or older, population density, or per capita GDP, which was consistent with that the outbreaks of COVID-19 in provinces outside Hubei were well managed and 
able to be controlled by the local health care systems. Thus, the differences in CFRs were likely due to epidemiological and clinical characteristics of the infected person. Other middle and high-income countries have showed similar outcomes. For example, Japan and Italy were both the countries with early outbreaks of COVID-19, while sharing a similar population age structure, however they appeared to have remarkable difference in CFR (3.32\% vs. $10.14 \%)$. Seriously aging population and the degree of mortality in elderly population may partly explain why Italy has such a high CFR, which may also be due to how COVID-19 related death is classified, as in Italy anyone who dies and has tested positive for the disease is reported as a COVID-19 related death. In addition, the number of people tested and how confirmed cases are defined also impacts this CFR. Subsequently, the true CFR of COVID-19 will not available until widespread testing and coherent definitions are congruently used throughout countries.

This study had several limitations. First, the sample size of this study was relatively small, with less than 200 deaths confirmed outside of Hubei province. Though the findings of this study may not be generalized to other populations, they do provide descriptive trends that can be useful for informing knowledge sharing practices and creating relevant, localized policies. Second, defined as the hospitalreported deaths among confirmed COVID-19 cases, the CFR in this study does not capture deaths among all COVID-19 patients (including asymptomatic or undiagnosed infections), which could lead to inaccuracy in estimating CFR. Further expansion of testing would help provide a more reliable denominator for estimating CFR, while modeling studies may supplement these existing shortcomings. Third, ecological bias in calculating the association using population index may be present. Lastly, as many additional environmental factors could affect the CFR, false negatives might be present considering the small sample size of our study. Continual review of daily reported diagnosis and mortality data should be carried out in order to better track, evaluate, and understand the evolving and unfolding epidemic.

\section{Conclusions}

Between January 22 and April 6, 2020, less than 200 deaths due to COVID-19 were reported outside of Hubei province, in mainland China, resulting in a CFR of $0.90 \%$. While this is much lower than in Hubei and other regions of the world, the results indicate that lower temperature, humidity and higher latitude may lead to increased risk of COVID-19-related death. Social environment factors were not found to be associated with CFR of COVID-19 in this study. Further monitoring of the prevalence of COVID-19 outside Hubei is needed in order to better inform guidelines and policies for prevention and control of the epidemic.

\section{Abbreviations}

COVID-19: the novel coronavirus disease 2019; CFR: case fatality rate; RT-PCR: real-time reversetranscriptase polymerase-chain-reaction; SD: standard deviation

\section{Declarations}




\section{Ethics approval and consent to participate}

The ethical approval or consent to participate was not applicable. Data were analyzed at aggregate level and no participants were contacted.

\section{Consent for publication}

Not Applicable.

\section{Availability of data and materials}

The COVID-19 data generated and/or analyzed are available in the National Health Commission of the People's Republic of China [http://www.nhc.gov.cn], and the data on environmental factors including natural environment factors and social environment factors were collected from the China National Statistical Yearbook [http://www.stats.gov.cn/tjsj/ndsj/2019/indexch.htm].

\section{Competing interests}

The authors have no conflicts of interest to declare.

\section{Funding}

This work was supported by the Xinglin Scholars Program of Chengdu University of Traditional Chinese Medicine [grant numbers XSGG2020006]; and Natural Science Foundation of Sichuan Collaborative Innovation Center of Elderly Care and Health [grant numbers YLZBZ2009].

\section{Authors' contributions}

$X F$ and $P D$ contributed equally. YF, SY and $Y C$ are joint correspondence authors. SY, $X F, P C$, and $Y C$ contributed to the study concept. PD, GL, PC, and JL contributed to the collection and assembly of data. $X F, X Y, D H$, and $X W$ contributed to the statistical analysis. SY, XF, PD, LY, and YC interpreted the findings and drafted the article. All the authors contributed to the critical revision of the article. SY, YF and YC are study guarantors. The corresponding authors attest that all listed authors meet authorship criteria and that no others meeting the criteria have been omitted.

\section{Acknowledgements}

We are extremely thankful to Sarah Robbins for her comments and revisions on earlier drafts of our paper.

\section{References}

1. Coronavirus disease 2019 (COVID-19): situation report-77. Published April 7, 2020. Accessed April 7, 2020. 
2. Health emergency office of China: The latest situation of COVID-19 outbreak. Published April 7, 2020. Accessed April 7, 2020.

DOl:http://www.nhc.gov.cn/xcs/yqtb/202004/4c63d42ef61142d6a3d827b9f9fd2fe9.shtml.

3. Guan W, Ni Z, Hu Y, et al. Clinical Characteristics of Coronavirus Disease 2019 in China[J]. The New England Journal of Medicine, 2020.

4. Tao Chen et al. Clinical characteristics of 113 deceased patients with coronavirus disease 2019: retrospective study.BMJ. 26 March 2020.

5. Li X, Wang L, Yan S, et al. Clinical characteristics of 25 death cases infected with COVID-19 pneumonia: a retrospective review of medical records in a single medical center, Wuhan, China[J]. medRxiv, 2020.

6. Reported by National Health Commission of the People's Republic of China. The 7th version of Chinese COVID-19 diagnosis and treatment plan. Accessed March 5, 2020.

DOI:http://www.nhc.gov.cn/yzygj/s7653p/202003/46c9294a7dfe4cef80dc7f5912eb1989.shtml

7. Compiled by National Bureau of Statistics of China. China Statistical Yearbook -2019. Beijing: China Statistics Press. Accessed March 20, 2020.

DOI: http://www.stats.gov.cn/tjsj/ndsj/2019/indexch.htm.

8. Li X, Wang L, Yan S, et al. Clinical characteristics of 25 death cases infected with COVID-19 pneumonia: a retrospective review of medical records in a single medical center, Wuhan, China[J]. medRxiv, 2020.

9. The Novel Coronavirus Pneumonia Emergency Response Epidemiology Team. The epidemiological characteristics of an outbreak of 2019 novel coronavirus diseases (COVID-19) in China. Chinese Journal of Epidemiology. 2020;41(2):145-151. DOI: 10.3760/cma.j.issn.0254-6450.2020.02.003.

10. Tao Chen, Di Wu, Huilong Chen, et al. Clinical characteristics of 113 deceased patients with coronavirus disease 2019: retrospective study. BMJ 2020;368:m1091.

DOI: https://doi.org/10.1136/bmj.m1091.

11. Epidemia COVID-19 Aggiornamento nazionale 19 marzo 2020-ore 16:00. Published March 20, 2020. Accessed March 28, 2020.

12. Actualización n57. Enfermedad por el coronavirus (COVID-19). Published March 27, 2020. Accessed March 28, 2020.

DOI:https://www.mallorcadiario.com/adjuntos/550842/Actualizacion_57_COVID-19.pdf.

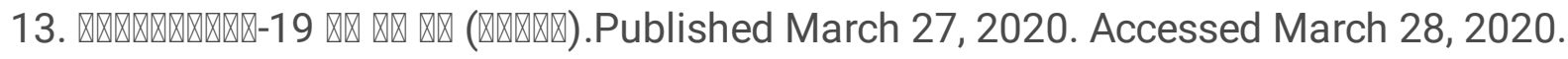


DOl:http://ncov.mohw.go.kr/upload/viewer/skin/doc.html?

fn=1585286935963_20200327142856.pdf\&rs=/upload/viewer/result/202003/.

14. Hu Shengshou, Gao Runlin, Liu Lisheng, et al. Summary of the 2018 Report on Cardiovascular Diseases in China. Chinese Circulation Journal, March, 2019, Vol. 34 No.3(Serial No.249).

15. Guoshuai Cai. Bulk and single-cell transcriptomics identify tobacco-use disparity in lung geneexpression of ACE2, the receptor of 2019-nCov. MedRxiv. Posted February 28, 2020.

16. Bicheng Zhang, Xiaoyang Zhou, Yanru Qiu, et al. Clinical characteristics of 82 death cases with COVID-19. MedRxiv. February 27, 2020.

17. Prince J, Wu Fan, Guo Yanfei et al. The burden of disease in older People and implications for health policy and practice [J]. Lancet, 2015, 385 (9967): 549 - 562.

18. Mohammad M Sajadi, Parham Habibzadeh, Augustin Vintzileos, et al. Temperature, Humidity and Latitude Analysis to Predict Potential Spread and Seasonality for COVID-19. SSRN, March 5, 2020.

\section{Tables}

Table1. The correlation between social environment factors and CFR

\begin{tabular}{|lll|}
\hline Object & Spearman r-value & $P$-value \\
\hline per capita GDP & -0.088 & 0.673 \\
\hline hospital beds per thousand population & -0.142 & 0.496 \\
\hline health technicians per thousand population & -0.235 & 0.257 \\
\hline medical practitioners per thousand population & -0.081 & 0.715 \\
\hline proportion of the population over the age of 65 & 0.042 & 0.848 \\
\hline population density & -0.017 & 0.941 \\
\hline
\end{tabular}

\section{Figures}


A

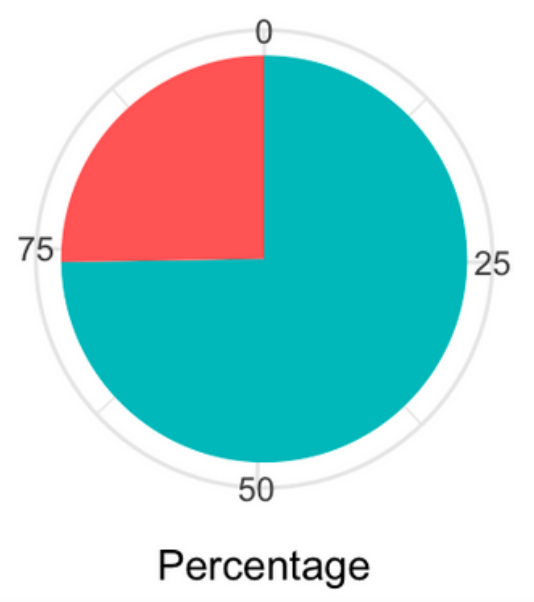

B

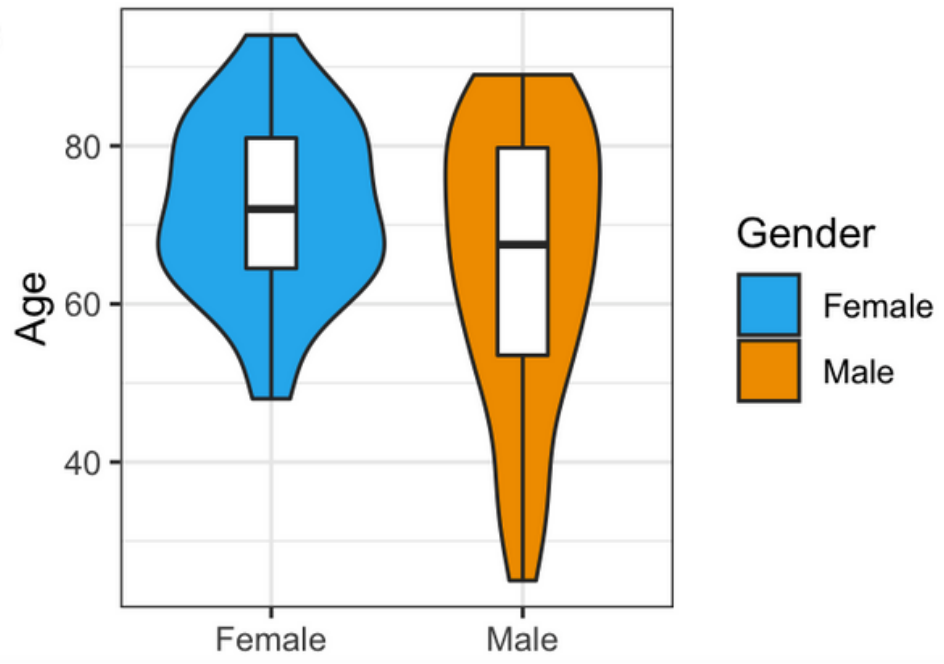

Figure 1

A. Proportion of deaths older than 65 years in the 99 deaths; B. Age distribution of 99 deaths by gender.
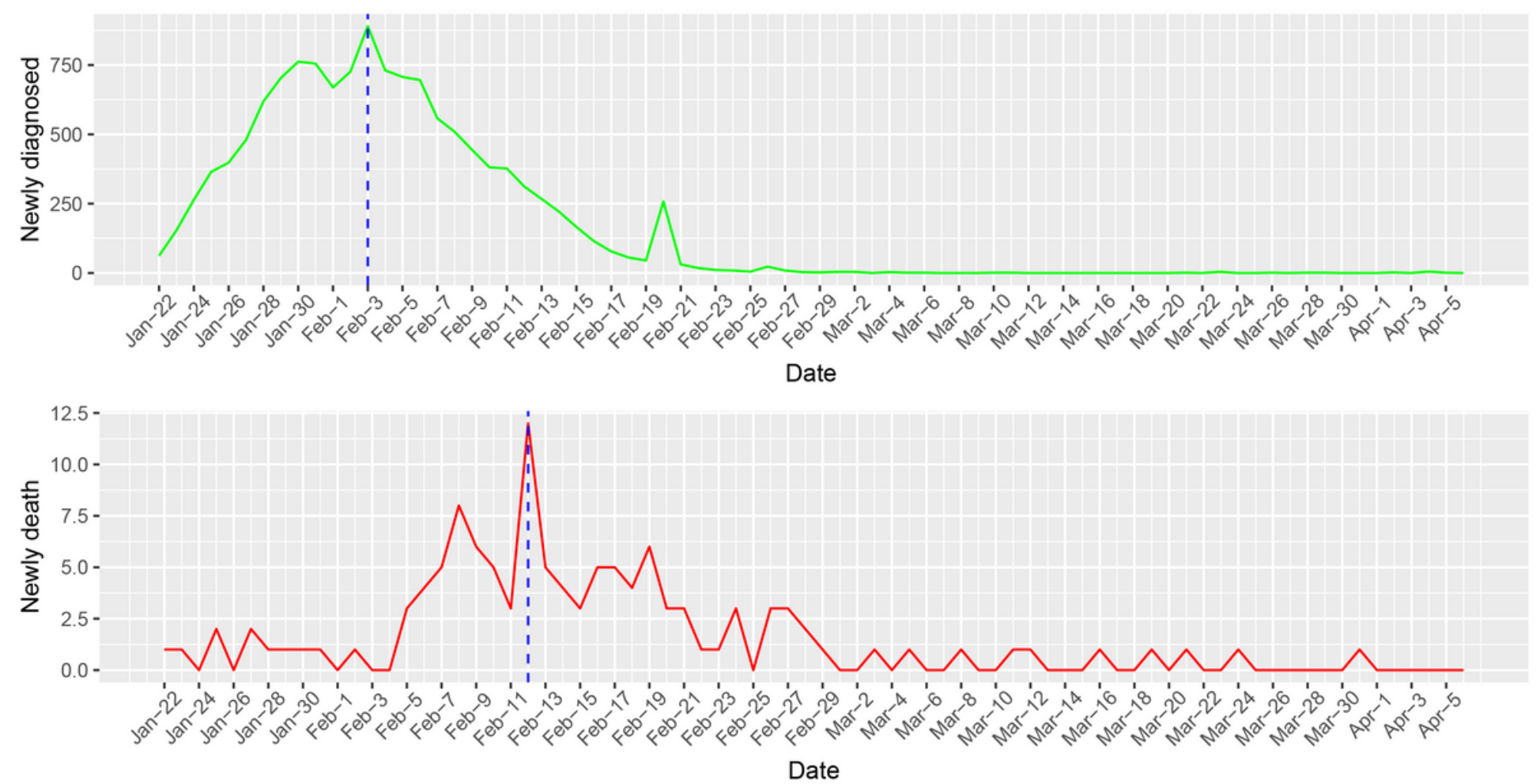

\section{Figure 2}

Daily new cases and daily new deaths due to COVID-19 between January 22 and April 5, 2020 outside of Hubei Province in Mainland China. The blue dotted lines indicate the peaks of daily new confirmed cases and daily new deaths, respectively. 


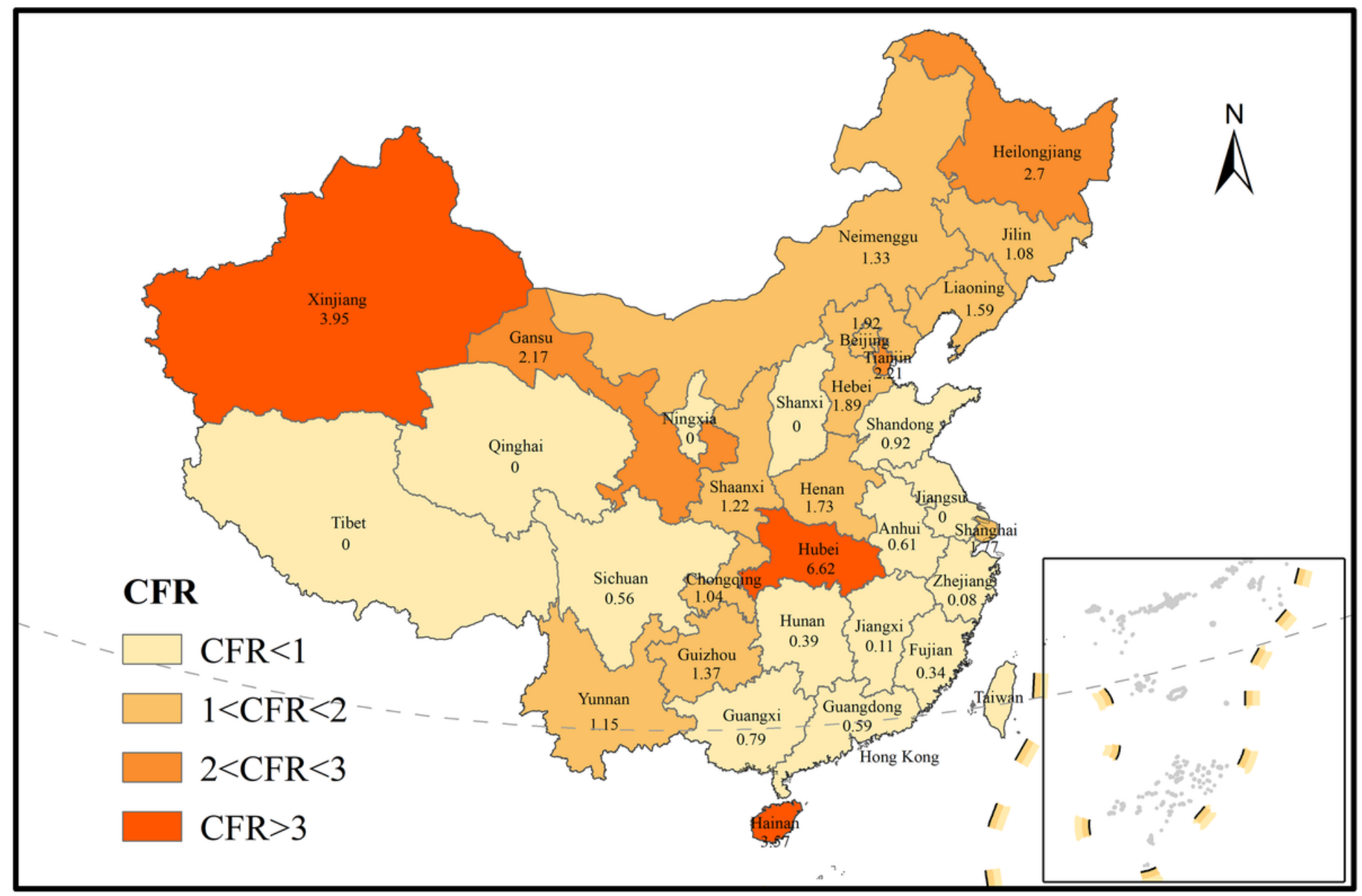

Figure 3

The COVID-19 CFR in different regions outside Hubei province, Chinese mainland between January 22 and April 6, 2020.
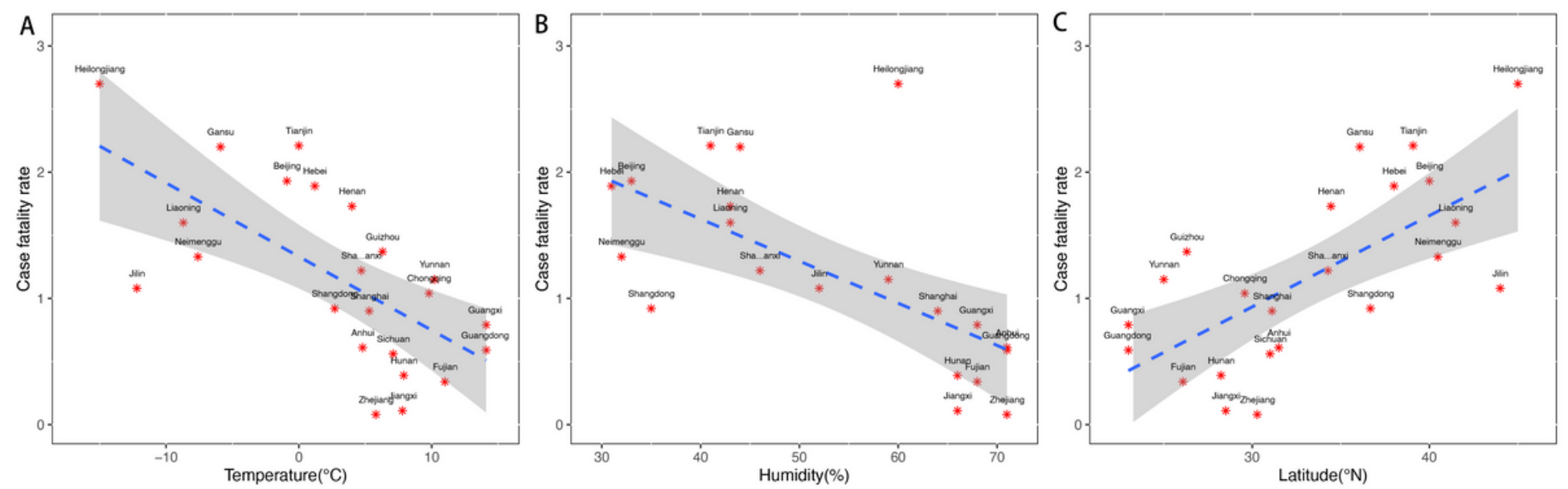

Figure 4

Scatter plot of the relationship between temperature, humidity, and latitude with CFR of COVID-19 cases in mainland China outside of Hubei province. The average temperature and humidity in the month of 
February 2020 was included, while the latitude of the provincial capital was considered. As seen in the Figure, both temperature and humidity were found to be negative correlated with an increasing number of reported deaths $(P<0.05)$, while latitude was positively correlated with CFR $(P=0.001)$. 